Bull. Austral. Math. Soc.

$11 \mathrm{R} 29,11 \mathrm{R} 20$

VOL. $70(2004) \quad$ [267-277]

\title{
THE CLASS GROUPS OF THE IMAGINARY ABELIAN NUMBER FIELDS WITH GALOIS GROUP $(\mathbb{Z} / 2 \mathbb{Z})^{n}$
}

\author{
JEOUNG-HWAN AHN AND SOUn-Hi Kwon
}

\begin{abstract}
Assuming the Generalised Riemann Hypothesis we determine all imaginary Abelian number fields $N$ whose Galois group $G(N / \mathbb{Q})$ is isomorphic to $(\mathbb{Z} / 2 \mathbb{Z})^{n}$ for some integers $n \geqslant 1$ and the square of every ideal of $N$ is principal.
\end{abstract}

For a finite group $G$ the smallest positive integer $m$ such that $g^{m}$ is the identity for all $g \in G$ is called the exponent of $G$ and denoted by $\exp (G)$. Chowla([6]) proved that there are finitely many imaginary quadratic number fields with class groups of exponents $\leqslant 2$. In $[24,16]$ it is shown that there are at most 66 such fields and that there are exactly 65 such fields under the assumption of the Generalised Riemann Hypothesis. These 65 fields are exactly the ones having one ideal class in each genus and are given in many sources in the literature, for example, $[2,8,20,5]$. K. Horie and M. Horie proved that there are only finitely many imaginary Abelian number fields of 2-power degrees with class groups of exponents $\leqslant 2$. (See $[13,14]$.) Many authors have studied the exponents of class groups of various number fields; for example, $[3,10,11,12,15,17,18,19,22]$. In particular, Louboutin determined all nonquadratic imaginary cyclic number fields of 2 -power degrees with class groups of exponents $\leqslant 2$. In this paper we show the following.

Theorem 1. Assume the Generalised Riemann Hypothesis. There are exactly $650(=65+483+99+3)$ imaginary Abelian number fields $N$ with Galois group isomorphic to $(\mathbb{Z} / 2 \mathbb{Z})^{n}$ for some integer $n$ and class group of exponent at most 2 . These fields are all of degree at most 16 with conductor at most 233905 and class number at most 32 . All of these fields of degrees $\geqslant 4$ over $\mathbb{Q}$ are compiled at the end of tnis paper.

For a number field $F$ let $\mathrm{Cl}(F), h_{F}, O_{F}, d_{F}$ denote the class group, the class number, the ring of algebraic integers, and the absolute value of the discriminant of $F$, respectively. If $F$ is a $C M$-field, then we let $F^{+}$and $h_{F}^{-}$denote its maximal totally real subfield and the relative class number of $F$, respectively. We let $f_{F}$ be the conductor of $F$ when $F$ is an Abelian number field.

Before proceeding to the proof of Theorem 1 we need the following result.

Received 22nd March, 2004

This research was supported by KOSEF GRANT R04-2001-00003-0.

Copyright Clearance Centre, Inc. Serial-fee code: 0004-9727/04 \$A2.00+0.00. 
Proposition 2. (a) Let $L / F$ be a quadratic extension of number fields. Assume that $\exp (\mathrm{Cl}(L)) \leqslant 2$. Then $\exp (\mathrm{Cl}(F))$ divides 4. In addition, the 4-rank of $\mathrm{Cl}(F)$ is at most one, and $\exp (\mathrm{Cl}(F)) \leqslant 2$ if $L / F$ is not unramified.

(b) Let $n \geqslant 2$ and let $m_{1}, m_{2}, \cdots, m_{n}$ be square free positive integers such that $N_{2^{n}}=\mathbb{Q}\left(\sqrt{-m_{1}}, \sqrt{-m_{2}}, \cdots, \sqrt{-m_{n}}\right)$ is an imaginary Abelian number fields of degree $2^{n}$ over $\mathbb{Q}$. Then $N_{2^{n}}$ has at least one imaginary subfield $M$ with $\left[N_{2^{n}}: M\right]=2$ such that $N_{2^{n}} / M$ is not unramified. Moreover, if $n \geqslant 3$, then $N_{2^{n}}$ has at least two distinct imaginary subfields $M$ and $M^{\prime}$ with $\left[N_{2^{n}}: M\right]=\left[N_{2^{n}}: M^{\prime}\right]=2$ such that neither the extension $N_{2^{n}} / M$ nor the extension $N_{2^{n}} / M^{\prime}$ is unramified.

Proof: (a) Let $\mathfrak{a}$ be an ideal of $O_{F}$. We have $\mathfrak{a}^{2} O_{L}=(\alpha)$ for some $\alpha \in O_{L}$ and $N_{L / F}\left(\mathfrak{a}^{2} O_{L}\right)=N_{L / F}(\alpha) O_{F}=\mathfrak{a}^{4}$, so $\exp (\mathrm{Cl}(F))$ divides 4 . For the second statement we use some well known results of class field theory. According to Takagi-Artin theorem if $L / F$ is unramified, then $\left|\mathrm{Cl}(F) / N_{L / F}(\mathrm{Cl}(L))\right|=[L: F]=2$ and so the 4-rank of $\mathrm{Cl}(F)$ can not exceed one, where $N_{L / F}$ denotes the norm map from $\mathrm{Cl}(L)$ to $\mathrm{Cl}(F)$. (See [4, Theorem 5.1, Chapter VII].) If $L / F$ is not unramified, then the norm map $N_{L / F}$ is surjective and so exp $(\mathrm{Cl}(F)) \leqslant 2$. (See [23, Theorem 10.1 and Theorem 5 in Appendix].)

(b) Let $S$ be the set of all imaginary subfields $M$ of $N_{2^{n}}$ with $\left[N_{2^{n}}: M\right]=2$. Let $p$ be a prime dividing $d_{N_{2^{n}}^{+}}$and let $\mathfrak{p}$ be a prime divisor of $p O_{N_{2^{n}}}$. Suppose that $N_{2^{n}} / M$ is unramified for all $M \in S$. Then the inertia field of $\mathfrak{p}$ would be equal to $N_{2^{n}}^{+}$. This contradicts the fact that $p \mid d_{N_{2^{n}}^{+}}$. For the second statement we take a field $M_{1} \in S$ such that $N_{2^{n}} / M_{1}$ is not unramified. Let $q$ be a prime dividing $d_{M_{1}^{+}}$(Since $n \geqslant 3$, we have $\left[M_{1}^{+}: \mathbb{Q}\right] \geqslant 2$ and $d_{M_{1}^{+}}>1$.) and $q$ a prime divisor of $q O_{N_{2^{n}}}$. Suppose that $N_{2^{n}} / M$ is unramified for all $M \in S \backslash\left\{M_{1}\right\}$. Then the inertia field of $q$ would be equal to either $M_{1}$ or $N_{2^{n}}^{+}$. Thus $q$ would be unramified in either $M_{1} / \mathbb{Q}$ or $N_{2^{n}}^{+} / \mathbb{Q}$. This contradicts the fact that $q \mid d_{M_{1}}$ and $q\left|d_{M_{1}^{+}}\right| d_{N_{2^{n}}^{+}}$.

Corollary 3. Let $N_{2^{n}}$ be as above.

(a) Assume that $\exp \left(\mathrm{Cl}\left(N_{4}\right)\right) \leqslant 2$. Then $N_{4}=k_{1} k_{2}$ is a compositum of two distinct imaginary quadratic fields $k_{1}$ and $k_{2}$ such that $\exp \left(\mathrm{Cl}\left(k_{1}\right)\right) \leqslant 2, \exp \left(\mathrm{Cl}\left(k_{2}\right)\right) \mid 4$ and at the same time the 4-rank of $\mathrm{Cl}\left(k_{2}\right)$ is at most one. Let $t$ be the number of distinct prime divisors of $d_{k_{2}}$. Assuming the Generalised Riemann Hypothesis we have either

(i) $\mathrm{Cl}\left(k_{2}\right) \cong(\mathbb{Z} / 2 \mathbb{Z})^{t-1}$ with $t \leqslant 7$ and $d_{k_{2}} \leqslant 1.4 \times 10^{6}$; or

(ii) $\mathrm{Cl}\left(k_{2}\right) \cong \mathbb{Z} / 4 \mathbb{Z} \times(\mathbb{Z} / 2 \mathbb{Z})^{t-2}$ with $t \leqslant 8$ and $d_{k_{2}} \leqslant 3.4 \times 10^{7}$.

(b) If $\exp \left(\mathrm{Cl}\left(N_{2^{n}}\right)\right) \leqslant 2$ with $n \geqslant 3$, then $N_{2^{n}}=M M^{\prime}$ is a compositum of two distinct imaginary subfields $M$ and $M^{\prime}$ such that $[M: \mathbb{Q}]=\left[M^{\prime}: \mathbb{Q}\right]=2^{n-1}$, $\exp (\mathrm{Cl}(M)) \leqslant 2$, and $\exp \left(\mathrm{Cl}\left(M^{\prime}\right)\right) \leqslant 2$.

Proof: The first statement of $(a)$, and $(b)$ follow from Proposition 2. We now prove the second statement of $(a)$. By [7, (18.3) Corollary] the 2-rank of $\mathrm{Cl}\left(k_{2}\right)$ is $t-1$. Let $k_{2}=\mathbb{Q}(\sqrt{-m})$ with the square free positive integer $m$. Assume $\mathrm{Cl}\left(k_{2}\right) \simeq(\mathbb{Z} / 2 \mathbb{Z})^{t-1}$. 
To determine upper bounds for $t$ and $d_{k_{2}}$ we divide our determinations into 12 cases according to the values $-m \bmod 4$ and the decompositions of the ideals (2) and (3) in $k_{2}$. Using Theorem 4 below we compute upper bounds for $t$ and $d_{k_{2}}$ in each case and take the worst upper bounds. We illustrate our method in the case that $-m \equiv 3 \bmod 4$ and (3) is ramified in $k_{2}$. Let $p_{i}$ be the $i$ th prime, that is, $p_{1}=2, p_{2}=3, p_{3}=5, \cdots$, et cetera. Set $f(t)=2 \prod_{i=1}^{t} p_{i}$ and $g(t)=\sqrt{f(t)} /(\ln f(t))$. Then $d_{k_{2}}=4 m=2 q_{1} q_{2} \cdots q_{t} \geqslant f(t)$, where $q_{i}$ 's are the distinct prime divisors of $d_{k_{2}}$. By Theorem 4 point (4) below

$$
h_{k_{2}}=2^{t-1} \geqslant \frac{2 \pi}{3 e} \frac{\sqrt{d_{k_{2}}}}{\ln d_{k_{2}}} \geqslant \frac{2 \pi}{3 e} \frac{\sqrt{f(t)}}{\ln f(t)}=\frac{2 \pi}{3 e} g(t),
$$

because $\sqrt{x} /(\ln x)$ is increasing for $x \geqslant e^{2}$. According to Bertrand's Postulate ([21, Theorem 8.7]) $p_{t}<p_{t+1}<2 p_{t}$. Hence

$$
\ln f(t+1)=\ln f(t)+\ln p_{t+1}<\ln f(t)+\ln 2 p_{t}<2 \ln f(t) .
$$

Therefore

$$
\frac{g(t+1)}{g(t)}=\frac{\ln f(t)}{\ln f(t+1)} \sqrt{p_{t+1}}>\frac{\sqrt{p_{t+1}}}{2} .
$$

Since $\left(\sqrt{p_{t+1}}\right) / 2>2$ for $t \geqslant 6, g(t)$ grows more rapidly than $2^{t-1}$ for $t \geqslant 6$. Note that $f(8)>f(7)>e^{2},(2 \pi / 3 e) g(8)=202.2304 \cdots>2^{8-1}=128$, but $(2 \pi / 3 e) g(7)$ $=56.2697 \cdots<2^{7-1}=64$. Therefore, $2^{t-1} \geqslant(2 \pi / 3 e) g(t)$ implies $t \leqslant 7$, and hence $h_{k_{2}} \leqslant 2^{6}$. The inequality $2^{6} \geqslant(2 \pi / 3 e)\left(\sqrt{d_{k_{2}}} / \ln d_{k_{2}}\right)$ yields $d_{k_{2}} \leqslant 1.4 \times 10^{6}$. This proves that if $-m \equiv 3 \bmod 4$ and (3) is ramified in $k_{2}$, then $t \leqslant 7$ and $d_{k_{2}} \leqslant 1.4 \times 10^{6}$. Similary we compute upper bounds for $t$ and $d_{k_{2}}$ in the remaining cases and verify that $t \leqslant 7$ and $d_{k_{2}} \leqslant 1.4 \times 10^{6}$ are the worst bounds. It follows that if $\mathrm{Cl}\left(k_{2}\right) \simeq(\mathbb{Z} / 2 \mathbb{Z})^{t-1}$, then $t \leqslant 7$ and $d_{k_{2}} \leqslant 1.4 \times 10^{6}$ (see [?, Theorem 2]). In a similar fashion we obtain that if $\mathrm{Cl}\left(k_{2}\right) \cong \mathbb{Z} / 4 \mathbb{Z} \times(\mathbb{Z} / 2 \mathbb{Z})^{t-2}$, then $t \leqslant 8$ and $d_{k_{2}} \leqslant 3.4 \times 10^{7}$. This completes the proof.

Theorem 4. ([16, Theorem 1]) Let $k$ be an imaginary quadratic field. Assuming the Generalised Riemann Hypothesis we have:

(1) $h_{k} \geqslant(\pi / 3 e)\left(\sqrt{d_{k}} / \ln d_{k}\right)$.

(2) When 2 is not inert in $k, h_{k} \geqslant(\pi / 2 e)\left(\sqrt{d_{k}} / \ln d_{k}\right)$.

(3) When 3 is not inert in $k, h_{k} \geqslant(4 \pi / 9 e)\left(\sqrt{d_{k}} / \ln d_{k}\right)$.

(4) When neither 2 nor 3 is inert in $k, h_{k} \geqslant(2 \pi / 3 e)\left(\sqrt{d_{k}} / \ln d_{k}\right)$ for $k$ with $d_{k} \neq 8$.

Proof of TheOREM 1: We verify that there are 65 imaginary quadratic fields $k_{1}$ with $\exp \left(\mathrm{Cl}\left(k_{1}\right)\right) \leqslant 2$ and 161 imaginary quadratic fields $k_{2}$ such that $\mathrm{Cl}\left(k_{2}\right)$ is isomorphic to $\mathbb{Z} / 4 \mathbb{Z} \times(\mathbb{Z} / 2 \mathbb{Z})^{t-2}$, where $t \leqslant 8$ was defined in Corollary 3 point $(a)$. We compute the relative class numbers of $12545(=65 \times((64 / 2)+161))$ quartic composita, and verify that the relative class numbers of all of these 12545 quartic composita are 
2-powers. Among those 12545 quartic fields only 9594 ones satisfy exp $\left(\mathrm{Cl}\left(N_{4}^{+}\right)\right) \leqslant 2$. For these 9594 quartic fields we compute $\mathrm{Cl}\left(N_{4}\right)$ and verify that there are exactly 483 fields $N_{4}$ with $\exp \left(\mathrm{Cl}\left(N_{4}\right)\right) \leqslant 2$. Our computational results are summerised in Tables 1 and 4 .

There are 12922 pairs of $\left(N_{4}^{(1)}, N_{4}^{(2)}\right)$ two distinct imaginary quartic fields such that $\exp \left(\mathrm{Cl}\left(N_{4}^{(i)}\right)\right) \leqslant 2$ for $i=1,2$ and $\left[N_{4}^{(1)} \cap N_{4}^{(2)}: \mathbb{Q}\right]=2$. Among those 12922 pairs 8686 ones give distinct composita $N_{8}=N_{4}^{(1)} N_{4}^{(2)}$. Among those 8686 composita $N_{8}=N_{4}^{(1)} N_{4}^{(2)}$ there are 172 octic fields $N_{8}$ such that every imaginary quartic subfield $K$ of $N_{8}$ satisfy that $\exp (\mathrm{Cl}(K)) \mid 4$ and at the same time the 4-rank of $\mathrm{Cl}(K)$ is at most 1. For those 172 octic fields we verify first whether both $h_{N_{\mathrm{B}}}^{-}$and $h_{N_{\mathrm{g}}^{+}}$are 2-powers, and then verify whether both $\mathrm{Cl}\left(\mathrm{N}_{8}^{+}\right)$and $\mathrm{Cl}\left(\mathrm{N}_{8}\right)$ are elementary 2-groups. Note that using the generalised Bernoulli numbers we can easily compute the relative class numbers of imaginary Abelian number fields. See Chapter 4 in [23]. There are 99 octic fields $N_{8}$ with exp $\left(\mathrm{Cl}\left(N_{8}\right)\right) \leqslant 2$. Our computational results are given in Tables 2 and 5.

In the same manner we take the composita $N_{16}=N_{8}^{(1)} N_{8}^{(2)}$ such that $\left[N_{8}^{(1)} \cap N_{8}^{(2)}\right.$ : $\mathbb{Q}]=4, \exp \left(\mathrm{Cl}\left(N_{8}^{(i)}\right)\right) \leqslant 2$ for $i=1,2$. We verify that there exist exactly three fields $N_{16}$ with $\exp \left(\mathrm{Cl}\left(N_{16}\right)\right) \leqslant 2$. Our computational results are given in Tables 3 and 6 .

The three composita of any two of those three fields $N_{16}$ with exp $\left(\mathrm{Cl}\left(N_{16}\right)\right) \leqslant 2$ are the same field ; $N_{32}=\mathbb{Q}(\sqrt{-1}, \sqrt{-2}, \sqrt{-3}, \sqrt{-5}, \sqrt{-7})$. Note that $N_{32}$ has a subfield $M=\mathbb{Q}(\sqrt{-1}, \sqrt{-2}, \sqrt{-5}, \sqrt{-7})$ such that the primes lying above 3 are ramified in the extension $N_{32} / M$. However, we verify that $\mathrm{Cl}(M)=\mathbb{Z} / 4 \mathbb{Z}$. By Proposition 2 point $(a)$, $\exp \left(\mathrm{Cl}\left(N_{32}\right)\right)>2$. Consequently, according to Proposition 2 point $(b)$ if $n \geqslant 5$, then $\exp \left(\mathrm{Cl}\left(N_{2^{n}}\right)\right)>2$. Our proof of Theorem 1 is now complete.

Our computations were carried out using PARI-GP ([1]) and KASH ([9]). Our source code is available to anyone interested by request.

Table 1

\begin{tabular}{|c|c|c|c|c|c|c|}
\hline $\mathrm{Cl}\left(N_{4}\right)$ & $(1)$ & $\mathbb{Z} / 2 \mathbb{Z}$ & $(\mathbb{Z} / 2 \mathbb{Z})^{2}$ & $(\mathbb{Z} / 2 \mathbb{Z})^{3}$ & $(\mathbb{Z} / 2 \mathbb{Z})^{4}$ & $(\mathbb{Z} / 2 \mathbb{Z})^{5}$ \\
\hline $\begin{array}{c}\text { number of fields } \\
N_{4}\end{array}$ & 47 & 160 & 112 & 132 & 26 & 6 \\
\hline $\begin{array}{c}\text { upper bounds for } \\
f_{N_{4}}\end{array}$ & 10921 & 65689 & 69601 & 233905 & 18204 & 142545 \\
\hline
\end{tabular}


Table 2

\begin{tabular}{|c|c|c|c|c|c|c|}
\hline $\mathrm{Cl}\left(N_{8}\right)$ & $(1)$ & $\mathbb{Z} / 2 \mathbb{Z}$ & $(\mathbb{Z} / 2 \mathbb{Z})^{2}$ & $(\mathbb{Z} / 2 \mathbb{Z})^{3}$ & $(\mathbb{Z} / 2 \mathbb{Z})^{4}$ & $(\mathbb{Z} / 2 \mathbb{Z})^{5}$ \\
\hline $\begin{array}{c}\text { number of fields } \\
N_{8}\end{array}$ & 17 & 27 & 19 & 32 & 1 & 3 \\
\hline $\begin{array}{c}\text { upper bounds for } \\
f_{N_{8}}\end{array}$ & 627 & 3553 & 760 & 5320 & 3315 & 6460 \\
\hline
\end{tabular}

Table 3

\begin{tabular}{|c|c|c|c|}
\hline $\mathrm{Cl}\left(N_{16}\right)$ & $(1)$ & $\mathbb{Z} / 2 \mathbb{Z}$ & $(\mathbb{Z} / 2 \mathbb{Z})^{2}$ \\
\hline $\begin{array}{c}\text { number of fields } \\
N_{16}\end{array}$ & 0 & 1 & 2 \\
\hline $\begin{array}{c}\text { upper bounds for } \\
f_{N_{16}}\end{array}$ & & 120 & 840 \\
\hline
\end{tabular}

Table 4

The fields $N_{4}=\mathbb{Q}\left(\sqrt{-m_{1}}, \sqrt{-m_{2}}\right)$ with $\exp \left(\mathrm{Cl}\left(N_{4}\right)\right) \leqslant 2$.

\begin{tabular}{|c|c|c|c|c|c|c|c|c|c|c|c|}
\hline$N_{r}$ & $\overrightarrow{m_{1}}$ & $m_{2}$ & $h_{N}^{+}$ & $\overline{h_{N_{4}}}$ & $f_{N_{4}}$ & $N+$. & $m_{1}$ & $m_{2}$ & $h_{N}+$ & $h_{N_{4}}$ & $f_{N_{4}}$ \\
\hline 1 & 1 & 2 & 1 & 1 & $2^{3}$ & 243 & 11 & 30 & 4 & 8 & $2^{3} \cdot 3 \cdot 5 \cdot 11$ \\
\hline 2 & 1 & 3 & 1 & 1 & $2^{2} \cdot 3$ & 244 & 2 & 165 & 4 & 16 & $2^{3} \cdot 3 \cdot 5 \cdot 11$ \\
\hline 3 & 3 & 15 & 1 & 1 & $3 \cdot 5$ & 245 & 5 & 67 & 2 & 2 & $2^{2} \cdot 5 \cdot 67$ \\
\hline 4 & 1 & 5 & 1 & 1 & $2^{2} \cdot 5$ & 246 & 11 & 123 & 2 & 2 & $3 \cdot 11 \cdot 41$ \\
\hline 5 & 3 & 7 & 1 & 1 & $3 \cdot 7$ & 247 & 115 & 345 & 1 & 8 & $2^{2} \cdot 3 \cdot 5 \cdot 23$ \\
\hline 6 & 2 & 3 & 1 & 1 & $2^{5} \cdot 3$ & 248 & 19 & 1387 & 1 & 2 & $19 \cdot 73$ \\
\hline 7 & 3 & 6 & 1 & $\overline{1}$ & $2^{3} \cdot 3$ & 249 & 6 & 177 & 1 & 4 & $2^{3} \cdot 3 \cdot 59$ \\
\hline 8 & 2 & 6 & 1 & 2 & $2^{x \cdot 3}$ & 250 & 2 & 177 & 2 & 8 & $2^{5} \cdot 3 \cdot 59$ \\
\hline 9 & 1 & 6 & 1 & 2 & $2^{3} \cdot 3$ & 251 & 1 & 357 & 2 & $\bar{B}$ & $2^{2} \cdot 3 \cdot 7 \cdot 17$ \\
\hline 10 & 1 & 7 & 1 & 1 & $2^{2} \cdot 7$ & 252 & 35 & 1435 & 1 & 4 & $5 \cdot 7 \cdot 41$ \\
\hline 11 & 3 & 11 & 1 & 1 & $3 \cdot 11$ & 253 & 3 & 1443 & 2 & $\bar{B}$ & $3 \cdot 13 \cdot 37$ \\
\hline 12 & 7 & 35 & 1 & 1 & $5 \cdot 7$ & 254 & 2 & 187 & 2 & 4 & $2^{3} \cdot 11 \cdot 17$ \\
\hline 13 & 3 & 39 & 1 & 2 & $3 \cdot 13$ & 255 & 35 & 43 & 2 & 2 & $5 \cdot 7 \cdot 43$ \\
\hline 14 & 2 & 10 & 1 & 1 & $2^{3} \cdot 5$ & 256 & 11 & 1507 & 1 & 2 & $11 \cdot 137$ \\
\hline 15 & 5 & 10 & 1 & 2 & $2^{3} \cdot 5$ & 257 & 35 & 385 & 1 & 8 & $2^{2} \cdot 5 \cdot 7 \cdot 11$ \\
\hline 16 & 2 & 5 & 2 & 2 & $2^{3} \cdot 5$ & 258 & 7 & 385 & 2 & 8 & $2^{2} \cdot 5 \cdot 7 \cdot 11$ \\
\hline 17 & 1 & 10 & 2 & 2 & $2^{3} \cdot 5$ & 259 & 11 & 385 & 2 & 8 & $2^{2} \cdot 5 \cdot 7 \cdot 11$ \\
\hline 18 & 1 & 11 & 1 & $t$ & $2^{2} \cdot 11$ & 260 & 3 & 130 & 4 & $\overline{\mathbf{B}}$ & $2^{5} \cdot 3 \cdot 5 \cdot 13$ \\
\hline 19 & 3 & 51 & 1 & 1 & $3 \cdot 17$ & 261 & 21 & 133 & 1 & 8 & $2^{2} \cdot 3 \cdot 7 \cdot 19$ \\
\hline 20 & 1 & 13 & 1 & 1 & $2^{2} \cdot 13$ & 262 & 21 & 57 & 1 & B & $2^{2} \cdot 3 \cdot 7 \cdot 19$ \\
\hline 21 & 11 & 53 & 1 & 2 & $3 \cdot 11$ & 263 & 6 & 67 & 2 & 2 & $2^{3} \cdot 3 \cdot 67$ \\
\hline 22 & 2 & 7 & 1 & $!$ & $2^{3} \cdot 7$ & 264 & 13 & 403 & 1 & 2 & $2^{2} \cdot 13 \cdot 31$ \\
\hline 23 & 7 & 14 & 1 & 2 & $2^{3} \cdot 7$ & 265 & 1 & 403 & 2 & 2 & $2^{2} \cdot 13 \cdot 31$ \\
\hline 24 & 3 & 19 & 1 & 1 & $3 \cdot 19$ & 266 & 7 & 58 & $\overline{2}$ & 4 & $2^{5} \cdot 7 \cdot 29$ \\
\hline 25 & 5 & 15 & 1 & 2 & $2^{2} \cdot 3 \cdot 5$ & 267 & 11 & 37 & 2 & 4 & $2^{2} \cdot 11 \cdot 37$ \\
\hline 26 & 1 & 15 & 2 & 2 & $2^{2} \cdot 3 \cdot 5$ & 268 & 15 & 1635 & 1 & 8 & $3 \cdot 5 \cdot 109$ \\
\hline 27 & 3 & 5 & 2 & 2 & $2^{2} \cdot 3 \cdot 5$ & 269 & 35 & 235 & 1 & 2 & $5 \cdot 7 \cdot 47$ \\
\hline 28 & 1 & $\overline{17}$ & $i$ & 2 & $2^{2} \cdot 17$ & 270 & 7 & 235 & 2 & 2 & $5 \cdot 7 \cdot 47$ \\
\hline 29 & 1 & 19 & 1 & $t$ & $2^{2} \cdot 19$ & 271 & 3 & 1659 & 1 & 4 & $3 \cdot 7 \cdot 79$ \\
\hline 30 & 7 & 11 & 1 & 1 & $7 \cdot 11$ & 272 & 19 & 22 & 2 & 2 & $2^{3} \cdot 11 \cdot 19$ \\
\hline
\end{tabular}


The fields $N_{4}=\mathbb{Q}\left(\sqrt{-m_{1}}, \sqrt{-m_{2}}\right)$ with
$\exp \left(\mathrm{Cl}\left(N_{4}\right)\right) \leqslant 2$.

\begin{tabular}{|c|c|c|c|c|c|c|c|c|c|c|c|}
\hline$N r$. & $m_{1}$ & $m_{2}$ & ${ }^{h} \mathrm{~N}_{4}^{+}$ & $h_{N_{4}}$ & $f_{N_{4}}$ & $\mathrm{Nr}$ & $m_{1}$ & $m_{2}$ & ${ }^{h} N_{t}$ & $h_{N_{4}}$ & $f_{N_{4}}$ \\
\hline 31 & 3 & 21 & 1 & 2 & $2^{2} \cdot 3 \cdot 7$ & 273 & 2 & 418 & 1 & 4 & $2^{3} \cdot 11 \cdot 1 \theta$ \\
\hline 32 & 1 & 21 & 1 & 2 & $2^{2} \cdot 3 \cdot 7$ & 274 & 10 & 43 & 2 & 2 & $2^{3} \cdot 5 \cdot 43$ \\
\hline 33 & 7 & 21 & 1 & 2 & $2^{2} \cdot 3 \cdot 7$ & 275 & 19 & 91 & 2 & 2 & $7 \cdot 13 \cdot 19$ \\
\hline 34 & 2 & 11 & 1 & 1 & $2^{3} \cdot 11$ & 276 & 1 & 435 & 4 & 8 & $2^{2} \cdot 3 \cdot 5 \cdot 29$ \\
\hline 35 & 11 & 22 & 1 & 1 & $2^{3} \cdot 11$ & 277 & 6 & 438 & 1 & $B$ & $2^{9} \cdot 3 \cdot 73$ \\
\hline 36 & 1 & 22 & 1 & 2 & $2^{3} \cdot 11$ & 278 & 7 & 1771 & 1 & 4 & $7 \cdot 11 \cdot 23$ \\
\hline 37 & 2 & 22 & 1 & 2 & $2^{3} \cdot 11$ & 279 & 5 & 445 & 1 & $B$ & $2^{2} \cdot 5 \cdot 89$ \\
\hline 38 & 7 & 91 & 1 & $I$ & $7 \cdot 13$ & 280 & 11 & 163 & 1 & 1 & $11 \cdot 163$ \\
\hline 39 & 2 & 13 & 2 & 2 & $2^{3} \cdot 13$ & 281 & 7 & 462 & 2 & 8 & $2^{3} \cdot 3 \cdot 7 \cdot 11$ \\
\hline 40 & 15 & $\overline{35}$ & 1 & $\overline{2}$ & $3 \cdot 5 \cdot 7$ & 282 & 21 & 462 & 1 & 16 & $2^{3} \cdot 3 \cdot 7 \cdot 11$ \\
\hline 41 & 7 & 15 & 2 & 2 & $3.5 \cdot 7$ & 283 & 42 & 462 & 1 & 16 & $2^{3} \cdot 3 \cdot 7 \cdot 11$ \\
\hline 42 & 3 & 35 & 2 & 2 & $3 \cdot 5 \cdot 7$ & 284 & 2 & 462 & 4 & 16 & $2^{3} \cdot 3 \cdot 7 \cdot 11$ \\
\hline 43 & 10 & 15 & 1 & 2 & $2^{3} \cdot 3 \cdot 5$ & 285 & 15 & 93 & 2 & 8 & $2^{2} \cdot 3 \cdot 5 \cdot 31$ \\
\hline 44 & 3 & 10 & 2 & 2 & $2^{3} \cdot 3 \cdot 5$ & 286 & 7 & 267 & 2 & 2 & 3.7 .89 \\
\hline 45 & 2 & 15 & 2 & 2 & $2^{3} \cdot 3 \cdot 5$ & 287 & 10 & 235 & 1 & 2 & $2^{3} \cdot 3 \cdot 47$ \\
\hline 46 & 5 & 30 & 1 & 4 & $2^{3} \cdot 3 \cdot 5$ & 288 & 2 & 233 & 2 & 2 & $2^{3} \cdot 5 \cdot 47$ \\
\hline 47 & 10 & 30 & 1 & 4 & $2^{3} \cdot 3 \cdot 5$ & 289 & 13 & 37 & 2 & 4 & $2^{2} \cdot 13 \cdot 37$ \\
\hline 48 & 1 & 30 & 2 & 4 & $2^{3} \cdot 3 \cdot 3$ & 290 & 21 & 483 & 1 & 8 & $2^{2} \cdot 3 \cdot 7 \cdot 23$ \\
\hline 49 & 2 & 30 & 2 & 4 & $2^{3} \cdot 3 \cdot 5$ & 291 & 11 & 1947 & 1 & 4 & $3 \cdot 11 \cdot 59$ \\
\hline 50 & 3 & 123 & 1 & 1 & 3.41 & 292 & 2 & 498 & 1 & 4 & $2^{3} \cdot 3 \cdot 83$ \\
\hline 51 & 3 & 43 & 1 & 1 & 3.43 & 293 & 15 & 1995 & 1 & 8 & $3 \cdot 5 \cdot 7 \cdot 19$ \\
\hline 52 & 1 & 33 & 1 & 2 & $2^{2} \cdot 3 \cdot 11$ & 294 & 3 & 1995 & 2 & 8 & $3 \cdot 5 \cdot 7 \cdot 19$ \\
\hline 53 & 11 & 33 & 1 & 2 & $2^{2} \cdot 3 \cdot 11$ & 295 & 19 & 1995 & 2 & 8 & $3.5 \cdot 7.19$ \\
\hline 54 & 3 & 33 & 1 & 2 & $2^{2} \cdot 3 \cdot 11$ & 296 & 22 & 253 & 1 & 4 & $2^{3} \cdot 11 \cdot 23$ \\
\hline 55 & 7 & 19 & 1 & 1 & $7 \cdot 18$ & 297 & 11 & 2035 & 2 & 8 & $5 \cdot 11 \cdot 37$ \\
\hline 56 & 2 & 34 & 1 & 2 & $2^{3} \cdot 17$ & 298 & 2 & 267 & 2 & 4 & $2^{3} \cdot 3 \cdot 89$ \\
\hline 57 & 5 & 35 & $\mathrm{I}$ & 2 & $2^{2} \cdot 5 \cdot 7$ & 299 & 11 & 195 & 4 & 8 & $3 \cdot 3 \cdot 11 \cdot 13$ \\
\hline 58 & 5 & 7 & 2 & 2 & $2^{2} \cdot 5 \cdot 7$ & 300 & 3 & 715 & 4 & 8 & $3 \cdot 5 \cdot 11 \cdot 13$ \\
\hline 59 & 1 & 35 & 2 & 2 & $2^{2} \cdot 5 \cdot 7$ & 301 & 3 & 2163 & 1 & 4 & $3 \cdot 7 \cdot 103$ \\
\hline 60 & 1 & 37 & 1 & 1 & $2^{2} \cdot 37$ & 302 & 6 & 91 & 4 & 8 & $2^{3} \cdot 3 \cdot 7 \cdot 13$ \\
\hline 61 & 2 & 19 & 1 & 1 & $2^{3} \cdot 19$ & 303 & 7 & 78 & 4 & 8 & $2^{3} \cdot 3 \cdot 7 \cdot 13$ \\
\hline 62 & 3 & 13 & 2 & 4 & $2^{2} \cdot 3 \cdot 13$ & 304 & 19 & 115 & 2 & 4 & 8.19 .23 \\
\hline 63 & 11 & 15 & 2 & 4 & $3 \cdot 5 \cdot 11$ & 305 & 43 & 51 & 2 & 4 & $3 \cdot 17 \cdot 43$ \\
\hline 64 & 3 & 42 & 1 & 2 & $2^{3} \cdot 3 \cdot 7$ & 306 & 37 & 555 & 2 & 8 & $2^{2} \cdot 3 \cdot 5 \cdot 37$ \\
\hline 65 & 2 & 42 & 1 & 2 & $2^{3} \cdot 3 \cdot 7$ & 307 & 1 & 555 & 4 & 8 & $2^{2} \cdot 3 \cdot 5 \cdot 37$ \\
\hline 66 & 7 & 42 & 1 & 2 & $2^{3} \cdot 3 \cdot 7$ & 308 & 13 & 43 & 2 & 4 & $2^{2} \cdot 13 \cdot 43$ \\
\hline 67 & 6 & 7 & 2 & 4 & $2^{3} \cdot 3 \cdot 7$ & 309 & 18 & 30 & 4 & 8 & $2^{3} \cdot 3 \cdot 5 \cdot 19$ \\
\hline 68 & 6 & 42 & 1 & 4 & $2^{3} \cdot 3 \cdot 7$ & 310 & 19 & 123 & 2 & 2 & $3 \cdot 19 \cdot 41$ \\
\hline 69 & 6 & 21 & 1 & 4 & $2^{3} \cdot 3 \cdot 7$ & 311 & 35 & 87 & 2 & 2 & $5 \cdot 7 \cdot 67$ \\
\hline 70 & 1 & 42 & 2 & 4 & $2^{3} \cdot 3 \cdot 7$ & 312 & 1 & 595 & 4 & 8 & $2^{2} \cdot 5 \cdot 7 \cdot 17$ \\
\hline 71 & 2 & 21 & 2 & 4 & $2^{3} \cdot 3 \cdot 7$ & 313 & 15 & 163 & 2 & 2 & $3 \cdot 5 \cdot 183$ \\
\hline 72 & 21 & 42 & 1 & 8 & $2^{3} \cdot 3 \cdot 7$ & 314 & 5 & 123 & 4 & 8 & $2^{2} \cdot 3 \cdot 5 \cdot 41$ \\
\hline 73 & 1 & 43 & 1 & 1 & $2^{2} \cdot 43$ & 315 & 11 & 38 & 2 & 2 & $2^{3} \cdot 11 \cdot 29$ \\
\hline 74 & 11 & 187 & 1 & 1 & $11 \cdot 17$ & 316 & 11 & 235 & 2 & 4 & 5.11 .47 \\
\hline 75 & 15 & 195 & 1 & 4 & $3 \cdot 5 \cdot 13$ & 317 & 21 & 93 & 1 & 8 & $2^{2} \cdot 3 \cdot 7 \cdot 31$ \\
\hline 76 & 3 & 195 & 2 & 4 & $3 \cdot 5 \cdot 13$ & 318 & 13 & 51 & 4 & 16 & $2^{2} \cdot 3 \cdot 13 \cdot 17$ \\
\hline 77 & 3 & 67 & 1 & 1 & 3.67 & 319 & 3 & 2687 & 1 & 4 & $3 \cdot 7 \cdot 127$ \\
\hline 78 & 7 & 203 & 1 & 2 & 7.29 & 320 & 10 & 67 & 2 & 2 & $2^{3} \cdot 5 \cdot 67$ \\
\hline 79 & $\mathrm{I}$ & 51 & 2 & 4 & $2^{2} \cdot 3 \cdot 17$ & 321 & 15 & 2715 & 1 & 8 & $3 \cdot 5 \cdot 181$ \\
\hline 80 & 11 & 19 & 1 & 1 & $11 \cdot 19$ & 322 & 30 & 345 & 1 & 18 & $2^{3} \cdot 3 \cdot 5 \cdot 23$ \\
\hline 81 & 3 & 219 & 1 & 2 & $3 \cdot 73$ & 323 & 19 & 37 & 2 & 2 & $2^{2} \cdot 19 \cdot 37$ \\
\hline 82 & 5 & 11 & 2 & 4 & $2^{2} \cdot 5 \cdot 11$ & 324 & 91 & 403 & 1 & 2 & $7 \cdot 13 \cdot 31$ \\
\hline 83 & 1 & 57 & 1 & 2 & $2^{2} \cdot 3 \cdot 19$ & 325 & 7 & 403 & 2 & 2 & $7 \cdot 13 \cdot 31$ \\
\hline B4 & 19 & $5 \overline{7}$ & 1 & 2 & $2^{2} \cdot 3 \cdot 19$ & 326 & 7 & 102 & 4 & 8 & $2^{3} \cdot 3 \cdot 7 \cdot 17$ \\
\hline B5 & 3 & 57 & 1 & 2 & $2^{2} \cdot 3 \cdot 10$ & 327 & 2 & 357 & 4 & 16 & $2^{3} \cdot 3 \cdot 7 \cdot 17$ \\
\hline 86 & 2 & 58 & 1 & 1 & $2^{3} \cdot 29$ & 328 & 5 & 715 & 2 & 8 & $2^{2} \cdot 5 \cdot 11 \cdot 13$ \\
\hline 87 & 1 & 58 & 2 & 2 & $2^{3} \cdot 29$ & 329 & 1 & 715 & 4 & 8 & $2^{2} \cdot 5 \cdot 11 \cdot 13$ \\
\hline 88 & 7 & 259 & 1 & 2 & $7 \cdot 37$ & $\mathbf{3 3 0}$ & 43 & 67 & 1 & 1 & 43.67 \\
\hline 89 & 5 & 13 & 2 & 4 & $2^{2} \cdot 5 \cdot 13$ & 331 & 11 & 267 & 2 & 4 & 3.11 .89 \\
\hline 90 & 6 & 22 & 1 & 2 & $2^{3} \cdot 3 \cdot 11$ & $\mathbf{3 3 2}$ & 7 & 742 & 2 & 8 & $2^{3} \cdot 7 \cdot 53$ \\
\hline 91 & 3 & 22 & 2 & 2 & $2^{3} \cdot 3 \cdot 11$ & $\mathbf{3 3 3}$ & 3 & 3003 & 2 & 8 & $3 \cdot 7 \cdot 11 \cdot 13$ \\
\hline 92 & 6 & 11 & 2 & 2 & $2^{3} \cdot 3 \cdot 11$ & 334 & 33 & 253 & 1 & 8 & $2^{2} \cdot 3 \cdot 11 \cdot 23$ \\
\hline 93 & 2 & 66 & 1 & 4 & $2^{3} \cdot 3 \cdot 11$ & 335 & 35 & 435 & 2 & 8 & $3 \cdot 5 \cdot 7 \cdot 29$ \\
\hline 94 & 6 & 33 & 1 & 4 & $2^{3} \cdot 3 \cdot 11$ & $\mathbf{3 3 6}$ & 7 & 435 & 4 & 8 & $3.5 \cdot 7.29$ \\
\hline 95 & 22 & 33 & 1 & 4 & $2^{3} \cdot 3 \cdot 11$ & 337 & 11 & 70 & 4 & 8 & $2^{3} \cdot 5 \cdot 7 \cdot 11$ \\
\hline 96 & 2 & 33 & 2 & 8 & $2^{3} \cdot 3 \cdot 11$ & 338 & 22 & 35 & 4 & 8 & $2^{3} \cdot 5 \cdot 7 \cdot 11$ \\
\hline 97 & 3 & 267 & 1 & 1 & 3.89 & 339 & 70 & 385 & 1 & 16 & $2^{3} \cdot 5 \cdot 7 \cdot 11$ \\
\hline 98 & $I$ & 67 & 1 & 1 & $2^{2}-67$ & 340 & 19 & 163 & 1 & 1 & $19 \cdot 163$ \\
\hline 99 & 3 & 91 & 2 & 4 & $3 \cdot 7 \cdot 13$ & 341 & 1 & 795 & 4 & 8 & $2^{2} \cdot 3 \cdot 5 \cdot 53$ \\
\hline 100 & 10 & 35 & 1 & 2 & $2^{3} \cdot 5 \cdot 7$ & 342 & 2 & 403 & 2 & 2 & $2^{3} \cdot 13 \cdot 31$ \\
\hline
\end{tabular}


The fields $N_{4}=\mathbb{Q}\left(\sqrt{-m_{1}}, \sqrt{-m_{2}}\right)$ with $\exp \left(\mathrm{Cl}\left(N_{4}\right)\right) \leqslant 2$.

\begin{tabular}{|c|c|c|c|c|c|c|c|c|c|c|c|}
\hline Nr. & $m_{1}$ & $m_{2}$ & $h_{\mathrm{N}}^{+}$ & $h_{N_{4}}$ & $f_{N_{4}}$ & Nr. & $m_{1}$ & $\overline{m_{2}}$ & $h_{N_{i}^{+}}$ & $h_{N_{4}}$ & $f_{N_{4}}$ \\
\hline 101 & 2 & 35 & 2 & 2 & $2^{3} \cdot 5 \cdot 7$ & 343 & 22 & 37 & 2 & 4 & $2^{3} \cdot 11 \cdot 37$ \\
\hline 102 & 7 & 10 & 2 & 2 & $2^{3} \cdot 5 \cdot 7$ & 344 & 5 & 163 & 2 & 2 & $2^{2} \cdot 5 \cdot 163$ \\
\hline 103 & 35 & 70 & 1 & 4 & $2^{3} \cdot 5 \cdot 7$ & 345 & 2 & 427 & 2 & 2 & $2^{3} \cdot 7 \cdot 61$ \\
\hline 104 & 7 & 70 & 2 & 4 & $2^{3} \cdot 5 \cdot 7$ & 346 & 31 & 67 & 2 & 4 & $3 \cdot 17 \cdot 67$ \\
\hline 105 & 10 & 70 & $l$ & 4 & $2^{3} \cdot 5 \cdot 7$ & 347 & 13 & 67 & 2 & 2 & $2^{2} \cdot 13 \cdot 67$ \\
\hline 106 & 5 & 70 & 1 & 4 & $2^{3} \cdot 5 \cdot 7$ & 348 & 19 & 187 & 2 & 4 & $11 \cdot 17 \cdot 19$ \\
\hline 107 & 1 & 70 & 2 & 4 & $2^{3} \cdot 5 \cdot 7$ & 349 & 19 & 195 & 4 & 8 & $3 \cdot 5 \cdot 13 \cdot 19$ \\
\hline 108 & 2 & 70 & 2 & 4 & $2^{3} \cdot 5 \cdot 7$ & 350 & 11 & 85 & 4 & 8 & $2^{2} \cdot 5 \cdot 11 \cdot 17$ \\
\hline 109 & 15 & 19 & 2 & 4 & $3 \cdot 5 \cdot 19$ & 351 & 5 & 187 & 4 & 8 & $2^{2} \cdot 5 \cdot 11 \cdot 17$ \\
\hline 110 & 3 & 291 & 1 & 2 & $3 \cdot 97$ & 352 & 22 & 43 & 2 & 2 & $2^{3} \cdot 11 \cdot 43$ \\
\hline 111 & 1 & 73 & 1 & 2 & $2^{2} \cdot 73$ & 353 & 11 & 3795 & $\overline{2}$ & 16 & $3 \cdot 5 \cdot 11 \cdot 23$ \\
\hline 112 & 2 & $\overline{37}$ & 2 & 2 & $2^{3} \cdot 37$ & 354 & 42 & 483 & 1 & 8 & $2^{3} \cdot 3 \cdot 7 \cdot 23$ \\
\hline 113 & 7 & 43 & 1 & 1 & $7 \cdot 43$ & 355 & 7 & 555 & 4 & 8 & $3 \cdot 5 \cdot 7 \cdot 37$ \\
\hline 114 & 3 & 73 & 1 & 4 & $2^{2} \cdot 7 \cdot 11$ & 356 & 6 & 163 & 2 & 2 & $2^{3} \cdot 3 \cdot 163$ \\
\hline 115 & 6 & 78 & $\mathbf{I}$ & 4 & $2^{3} \cdot 3 \cdot 13$ & 357 & 43 & 91 & 2 & 4 & $7 \cdot 13 \cdot 43$ \\
\hline 116 & 3 & 78 & 2 & 4 & $2^{3} \cdot 3 \cdot 13$ & 358 & 19 & 58 & 2 & 2 & $2^{3} \cdot 19 \cdot 29$ \\
\hline 117 & 13 & 78 & 1 & 4 & $2^{3} \cdot 3 \cdot 13$ & 359 & 13 & 85 & 4 & 16 & $2^{2} \cdot 5 \cdot 13 \cdot 17$ \\
\hline 118 & 1 & 78 & 2 & 4 & $2^{3} \cdot 3 \cdot 13$ & 360 & 11 & 403 & 2 & 2 & $11 \cdot 13 \cdot 31$ \\
\hline 119 & 2 & 78 & 2 & 4 & $2^{3} \cdot 3 \cdot 13$ & 361 & 19 & 235 & 2 & 4 & $5 \cdot 19 \cdot 47$ \\
\hline 120 & 6 & 13 & 2 & 4 & $2^{3} \cdot 3 \cdot 13$ & 362 & 2 & 1122 & 2 & 16 & $2^{3} \cdot 3 \cdot 11 \cdot 17$ \\
\hline 121 & 19 & 323 & 1 & 2 & $17 \cdot 19$ & 363 & 11 & 427 & 2 & 2 & $7 \cdot 11 \cdot 61$ \\
\hline 122 & 2 & 82 & 1 & 2 & $2^{3} \cdot 4 !$ & 364 & 2 & 595 & 1 & 8 & $2^{3} \cdot 5 \cdot 7 \cdot 17$ \\
\hline 123 & 5 & 85 & 1 & 4 & $2^{2} \cdot 5 \cdot 17$ & 365 & 11 & 435 & 4 & 8 & $3 \cdot 5 \cdot 11 \cdot 29$ \\
\hline 124 & 1 & 85 & 2 & 4 & $2^{2} \cdot 5 \cdot 17$ & 366 & 10 & 123 & 4 & 8 & $2^{5} \cdot 3 \cdot 5 \cdot 41$ \\
\hline 125 & 2 & 43 & 1 & 1 & $2^{3} \cdot 43$ & 367 & 43 & 115 & 2 & 2 & $5 \cdot 23 \cdot 43$ \\
\hline 126 & 15 & 115 & 1 & 2 & $3 \cdot 5 \cdot 23$ & 368 & 2 & 627 & 4 & 16 & $2^{3} \cdot 3 \cdot 11 \cdot 19$ \\
\hline 127 & 3 & 115 & 2 & 2 & $3 \cdot 5 \cdot 23$ & 369 & 11 & 5115 & 2 & 16 & $3 \cdot 5 \cdot 11 \cdot 31$ \\
\hline 128 & 7 & 51 & 2 & 2 & $3 \cdot 7 \cdot 17$ & 370 & 3 & 5187 & 2 & 16 & $3 \cdot 7 \cdot 13 \cdot 19$ \\
\hline 129 & 13 & 81 & 1 & 2 & $2^{7} \cdot 7 \cdot 13$ & 371 & 6 & 1302 & 1 & 16 & $2^{3} \cdot 3 \cdot 7 \cdot 31$ \\
\hline 130 & 1 & 91 & 2 & 2 & $2^{2} \cdot 7 \cdot 13$ & 372 & 43 & 123 & 2 & 4 & $3.41 \cdot 43$ \\
\hline 131 & 7 & 13 & 2 & 2 & $2^{2} \cdot 7 \cdot 13$ & 373 & 13 & 102 & 4 & 16 & $2^{5} \cdot 3 \cdot 13 \cdot 17$ \\
\hline 132 & 3 & 93 & 1 & 2 & $2^{2} \cdot 3 \cdot 31$ & 374 & 11 & 483 & 4 & 8 & $3 \cdot 7 \cdot 11 \cdot 23$ \\
\hline 133 & 1 & 93 & 1 & 2 & $2^{2} \cdot 3 \cdot 31$ & 375 & 19 & 70 & 4 & 8 & $2^{3} \cdot 5 \cdot 7 \cdot 19$ \\
\hline 134 & 5 & 19 & 2 & 4 & $2^{2} \cdot 5 \cdot 19$ & 376 & 7 & 190 & 4 & 8 & $2^{2} \cdot 5 \cdot 7 \cdot 19$ \\
\hline 135 & 11 & 35 & 2 & 4 & $3 \cdot 7 \cdot 11$ & 377 & 5 & 267 & 4 & 8 & $2^{2} \cdot 3 \cdot 5 \cdot 89$ \\
\hline 136 & 1 & 97 & 1 & 2 & $2^{2} \cdot 97$ & 378 & 115 & 235 & 1 & 2 & $5 \cdot 23 \cdot 47$ \\
\hline 137 & 51 & 102 & 1 & 4 & $2^{3} \cdot 3 \cdot 17$ & 379 & 7 & 5467 & 1 & 4 & $7 \cdot 11 \cdot 71$ \\
\hline 138 & 6 & 102 & 1 & 4 & $2^{5} \cdot 3 \cdot 17$ & 380 & 7 & 795 & 4 & 8 & $3 \cdot 5 \cdot 7 \cdot 53$ \\
\hline 139 & 2 & 51 & 2 & 4 & $2^{3} \cdot 3 \cdot 17$ & 381 & 35 & 163 & 2 & 2 & $5 \cdot 7 \cdot 163$ \\
\hline 140 & 1 & 102 & 2 & 8 & $2^{3} \cdot 3 \cdot 17$ & 382 & 11 & 130 & 4 & 8 & $2^{3} \cdot 5 \cdot 11 \cdot 13$ \\
\hline 141 & 2 & 102 & 2 & 8 & $2^{5} \cdot 3 \cdot 17$ & 383 & 5 & 1435 & 2 & 8 & $2^{2} \cdot 5 \cdot 7 \cdot 41$ \\
\hline 142 & 7 & 427 & 1 & 1 & $7 \cdot 61$ & 384 & 1 & 1435 & 4 & 8 & $2^{2} \cdot 5 \cdot 7 \cdot 41$ \\
\hline 143 & 15 & 435 & 1 & 4 & $3 \cdot 5 \cdot 29$ & 385 & 22 & 67 & 2 & 2 & $2^{5} \cdot 11 \cdot 67$ \\
\hline 144 & 10 & 11 & 2 & 4 & $2^{3} \cdot 5 \cdot 11$ & 386 & 15 & 403 & 4 & 8 & $3 \cdot 5 \cdot 13 \cdot 31$ \\
\hline 145 & 10 & 22 & 2 & 4 & $2^{3} \cdot 5 \cdot 11$ & 387 & 11 & 555 & 4 & 16 & $3 \cdot 5 \cdot 11 \cdot 37$ \\
\hline 146 & 5 & 22 & 2 & 4 & $2^{3} \cdot 5 \cdot 11$ & 388 & 37 & 43 & 2 & 2 & $2^{2} \cdot 37 \cdot 43$ \\
\hline 147 & 3 & 37 & 2 & 4 & $2^{2} \cdot 3 \cdot 37$ & 389 & 15 & 427 & 4 & 8 & $3 \cdot 5 \cdot 7 \cdot 61$ \\
\hline 148 & 6 & 19 & 2 & 2 & $2^{3} \cdot 3 \cdot 19$ & 390 & 19 & 85 & 4 & 16 & $2^{2} \cdot 5 \cdot 17 \cdot 19$ \\
\hline 149 & 2 & 114 & 1 & 4 & $2^{3} \cdot 3 \cdot 19$ & 391 & 10 & 163 & 2 & 2 & $2^{5} \cdot 5 \cdot 163$ \\
\hline 150 & 6 & 57 & 1 & 4 & $2^{3} \cdot 3 \cdot 19$ & 392 & 11 & 595 & 4 & 8 & $5 \cdot 7 \cdot 11 \cdot 17$ \\
\hline 151 & 2 & 57 & 2 & 8 & $2^{3} \cdot 3 \cdot 19$ & 393 & 43 & 163 & 1 & 1 & $43 \cdot 163$ \\
\hline 152 & 5 & 115 & 1 & 2 & $2^{2} \cdot 5 \cdot 23$ & 394 & 57 & 93 & 1 & 8 & $2^{2} \cdot 3 \cdot 19 \cdot 31$ \\
\hline 153 & 1 & 115 & 2 & 2 & $2^{2} \cdot 5 \cdot 23$ & 395 & 19 & 7315 & 2 & 16 & $5 \cdot 7 \cdot 11 \cdot 19$ \\
\hline 154 & 7 & 483 & $!$ & 2 & $3 \cdot 7 \cdot 23$ & 396 & 10 & 187 & 4 & 8 & $2^{3} \cdot 5 \cdot 11 \cdot 17$ \\
\hline 155 & 3 & 483 & 1 & 2 & $3 \cdot 7 \cdot 23$ & 397 & 37 & 51 & 4 & $B$ & $2^{2} \cdot 3 \cdot 17 \cdot 37$ \\
\hline 156 & 3 & 163 & 1 & 1 & $3 \cdot 163$ & 398 & 19 & 403 & 2 & 2 & $13 \cdot 19 \cdot 31$ \\
\hline 157 & 1 & 123 & 2 & 4 & $2^{2} \cdot 3 \cdot 41$ & 399 & 19 & 102 & 4 & 8 & $2^{3} \cdot 3 \cdot 17 \cdot 19$ \\
\hline 158 & 1 & 130 & 4 & 8 & $2^{3} \cdot 5 \cdot 13$ & 400 & 11 & 7755 & 2 & 16 & $3 \cdot 5 \cdot 11 \cdot 47$ \\
\hline 159 & 1 & 133 & 1 & 2 & $2^{2} \cdot 7 \cdot 19$ & 401 & 33 & 177 & 1 & 8 & $2^{2} \cdot 3 \cdot 11 \cdot 59$ \\
\hline 160 & 7 & 133 & 1 & 2 & $2^{2} \cdot 7 \cdot 19$ & 402 & 43 & 187 & 2 & 4 & $11 \cdot 17 \cdot 43$ \\
\hline 161 & 19 & 133 & 1 & 2 & $2^{2} \cdot 7 \cdot 19$ & 403 & 35 & 58 & 4 & 8 & $2^{3} \cdot 5 \cdot 7 \cdot 2 \theta$ \\
\hline 162 & 2 & 67 & 1 & 1 & $2^{3} \cdot 67$ & 104 & 67 & 123 & 2 & 2 & $3 \cdot 41 \cdot 67$ \\
\hline 163 & 15 & 555 & 1 & 4 & $3 \cdot 5 \cdot 37$ & 405 & 19 & 435 & 4 & 8 & $3 \cdot 5 \cdot 19 \cdot 29$ \\
\hline 164 & 3 & 555 & 2 & 4 & $3 \cdot 5 \cdot 37$ & 406 & 51 & 163 & 2 & 2 & $3 \cdot 17 \cdot 163$ \\
\hline 165 & 51 & 187 & 1 & 2 & $3 \cdot 11 \cdot 17$ & 407 & 11 & 190 & 4 & 8 & $2^{3} \cdot 5 \cdot 11 \cdot 19$ \\
\hline 166 & 3 & 187 & 2 & 2 & $3 \cdot 11 \cdot 17$ & 408 & 43 & 195 & 4 & 8 & $3 \cdot 5 \cdot 13 \cdot 43$ \\
\hline 167 & 11 & 51 & 2 & 2 & $3 \cdot 11 \cdot 17$ & 409 & 13 & 163 & 2 & 2 & $2^{2} \cdot 13 \cdot 163$ \\
\hline 168 & 11 & 13 & 2 & 2 & $2^{2} \cdot 11 \cdot 13$ & 410 & 15 & 8835 & 1 & 16 & $3 \cdot 5 \cdot 19 \cdot 31$ \\
\hline 169 & 7 & 154 & 1 & 4 & $2^{3} \cdot 7 \cdot 11$ & 411 & 7 & 2233 & 2 & 16 & $2^{2} \cdot 7 \cdot 11 \cdot 29$ \\
\hline 170 & 7 & 22 & 2 & 4 & $2^{3} \cdot 7 \cdot 11$ & 412 & 19 & 483 & 4 & 8 & $3 \cdot 7 \cdot 19 \cdot 23$ \\
\hline
\end{tabular}


The fields $N_{4}=\mathbb{Q}\left(\sqrt{-m_{1}}, \sqrt{-m_{2}}\right)$ with $\exp \left(\mathrm{Cl}\left(N_{4}\right)\right) \leqslant 2$.

\begin{tabular}{|c|c|c|c|c|c|c|c|c|c|c|c|}
\hline Nr. & $m_{1}$ & $\overline{m_{2}}$ & $\overline{h_{N}+}$ & $h_{N_{4}}$ & $f N_{4}$ & $N r$. & $\overline{m_{t}}$ & $\overline{m_{2}}$ & $h_{N+}$ & $h_{N_{4}}$ & $/ N_{4}$ \\
\hline 171 & 3 & 627 & 1 & 2 & $3 \cdot 11 \cdot 19$ & 413 & 6 & 403 & 1 & 8 & $2^{3} \cdot 3 \cdot 13 \cdot 31$ \\
\hline 172 & 11 & 627 & 1 & 2 & $3 \cdot 11 \cdot 19$ & 414 & 13 & 187 & 4 & 8 & $2^{2} \cdot 11 \cdot 13 \cdot 17$ \\
\hline 173 & 19 & 627 & 1 & 2 & $3 \cdot 11 \cdot 19$ & 415 & 19 & 130 & 4 & 8 & $2^{3} \cdot 5 \cdot 13 \cdot 19$ \\
\hline 174 & 15 & 43 & 2 & 2 & $3 \cdot 5 \cdot 43$ & 416 & 37 & 67 & 2 & 4 & $2^{2} \cdot 37 \cdot 67$ \\
\hline 175 & 3 & 651 & 1 & 4 & $3 \cdot 7 \cdot 31$ & 417 & 43 & 58 & 2 & 2 & $2^{3} \cdot 29 \cdot 43$ \\
\hline 176 & 1 & 163 & 1 & 1 & $2^{2} \cdot 163$ & 418 & 115 & 435 & 2 & 8 & $3 \cdot 5 \cdot 23 \cdot 29$ \\
\hline 177 & 11 & 165 & 2 & 8 & $2^{2} \cdot 3 \cdot 5 \cdot 11$ & 419 & 43 & 235 & 2 & 2 & $5 \cdot 43 \cdot 47$ \\
\hline 178 & 19 & 35 & 2 & 4 & $5 \cdot 7 \cdot 19$ & 420 & 22 & 115 & 4 & 8 & $2^{3} \cdot 5 \cdot 11 \cdot 23$ \\
\hline 179 & 2 & 85 & 4 & 8 & $2^{3} \cdot 5 \cdot 17$ & 421 & 6 & 427 & 4 & 8 & $2^{3} \cdot 3 \cdot 7 \cdot 61$ \\
\hline 180 & 3 & 58 & 2 & 2 & $2^{3} \cdot 3 \cdot 29$ & 422 & 19 & 555 & 4 & 8 & $3 \cdot 5 \cdot 19 \cdot 37$ \\
\hline 181 & 15 & 235 & 1 & 2 & $3 \cdot 5 \cdot 47$ & 423 & 10 & 267 & 4 & 8 & $2^{3} \cdot 3 \cdot 5 \cdot 89$ \\
\hline 182 & 3 & 235 & 2 & 2 & $3 \cdot 5 \cdot 47$ & 424 & 67 & 163 & 1 & 1 & $67 \cdot 163$ \\
\hline 183 & 1 & 177 & 1 & 2 & $2^{2} \cdot 3 \cdot 59$ & 425 & 19 & 595 & 4 & 16 & $5 \cdot 7 \cdot 17 \cdot 19$ \\
\hline 184 & 3 & 177 & 1 & 2 & $2^{2} \cdot 3 \cdot 59$ & 426 & 2 & 1435 & 4 & 8 & $2^{3} \cdot 5 \cdot 7 \cdot 41$ \\
\hline 185 & 11 & 715 & 2 & 4 & $5 \cdot 11 \cdot 13$ & 427 & 43 & 267 & 2 & 2 & $3 \cdot 43 \cdot 89$ \\
\hline 186 & 3 & 723 & 1 & 2 & $3 \cdot 241$ & $\overline{428}$ & 11 & 11715 & 2 & 16 & $3 \cdot 5 \cdot 11 \cdot 71$ \\
\hline 187 & 2 & 91 & 2 & 2 & $2^{3} \cdot 7 \cdot 13$ & 429 & 67 & 187 & 2 & 4 & $11 \cdot 17 \cdot 67$ \\
\hline 188 & 11 & 67 & 1 & 1 & $11 \cdot 67$ & 430 & 13 & 3315 & 4 & 32 & $2^{2} \cdot 3 \cdot 5 \cdot 13 \cdot 17$ \\
\hline 189 & 5 & 37 & 2 & 4 & $2^{2} \cdot 5 \cdot 37$ & 431 & 1 & 3315 & 8 & 32 & $2^{2} \cdot 3 \cdot 5 \cdot 13 \cdot 17$ \\
\hline 190 & 6 & 93 & 1 & 4 & $2^{3} \cdot 3 \cdot 31$ & 432 & 43 & 78 & 4 & 8 & $2^{5} \cdot 3 \cdot 13 \cdot 43$ \\
\hline 191 & 2 & 93 & 2 & 4 & $2^{3} \cdot 3 \cdot 31$ & 433 & 19 & 715 & 4 & 8 & $5 \cdot 11 \cdot 13 \cdot 19$ \\
\hline 192 & 1 & 187 & 2 & 4 & $2^{2} \cdot 11 \cdot 17$ & 434 & 13 & 267 & 4 & 8 & $2^{2} \cdot 3 \cdot 13 \cdot 89$ \\
\hline 193 & 19 & 190 & 2 & 4 & $2^{3} \cdot 5 \cdot 19$ & 435 & 115 & 123 & 4 & 8 & $3 \cdot 5 \cdot 23 \cdot 41$ \\
\hline 194 & 10 & 19 & 2 & 4 & $2^{3} \cdot 5 \cdot 19$ & 436 & 22 & 163 & 2 & 2 & $2^{3} \cdot 11 \cdot 163$ \\
\hline 195 & 5 & 190 & 1 & 4 & $2^{3} \cdot 5 \cdot 19$ & 437 & 43 & 85 & 4 & 8 & $2^{2} \cdot 5 \cdot 17 \cdot 43$ \\
\hline 196 & 10 & 190 & 1 & 4 & $2^{3} \cdot 5 \cdot 19$ & 438 & 3 & 14763 & 2 & 16 & $3 \cdot 7 \cdot 19 \cdot 37$ \\
\hline 197 & 1 & 190 & 2 & 4 & $2^{3} \cdot 5 \cdot 19$ & 439 & 91 & 163 & 2 & 2 & $7 \cdot 13 \cdot 163$ \\
\hline 198 & 2 & 190 & 2 & 4 & $2^{3} \cdot 5 \cdot 19$ & 440 & 19 & 795 & 4 & 8 & $3 \cdot 5 \cdot 19 \cdot 53$ \\
\hline 199 & 7 & 763 & 1 & 2 & $7 \cdot 109$ & 441 & 58 & 67 & 2 & 4 & $2^{3} \cdot 29 \cdot 67$ \\
\hline 200 & 1 & 193 & 1 & 2 & $2^{2} \cdot 193$ & 442 & 67 & 235 & 2 & 2 & $5 \cdot 47 \cdot 67$ \\
\hline 201 & 13 & 195 & 2 & 8 & $2^{2} \cdot 3 \cdot 5 \cdot 13$ & 443 & 11 & 1435 & 4 & 8 & $5 \cdot 7 \cdot 11 \cdot 41$ \\
\hline 202 & 1 & 195 & 4 & 8 & $2^{2} \cdot 3 \cdot 5 \cdot 13$ & 444 & 43 & 403 & 2 & 4 & $13 \cdot 31 \cdot 43$ \\
\hline 203 & 35 & 115 & 1 & 2 & $5 \cdot 7 \cdot 23$ & 445 & 43 & 102 & 4 & 8 & $2^{5} \cdot 3 \cdot 17 \cdot 43$ \\
\hline 204 & 7 & 115 & 2 & 2 & $5 \cdot 7 \cdot 23$ & 446 & 37 & 123 & 4 & 16 & $2^{x} \cdot 3 \cdot 37 \cdot 41$ \\
\hline 205 & 5 & 205 & 1 & 8 & $2^{2} \cdot 5 \cdot 41$ & 447 & 43 & 427 & 2 & 2 & $7 \cdot 43 \cdot 61$ \\
\hline 206 & 7 & 30 & 4 & 8 & $2^{3} \cdot 3 \cdot 5 \cdot 7$ & 448 & 115 & 163 & 2 & 2 & $5 \cdot 23 \cdot 163$ \\
\hline 207 & 5 & 43 & 2 & 2 & $2^{2} \cdot 5 \cdot 43$ & 449 & 123 & 163 & 2 & 4 & $3 \cdot 41 \cdot 163$ \\
\hline 208 & 7 & 123 & 2 & 2 & $3 \cdot 7 \cdot 41$ & 450 & 51 & 403 & 4 & 8 & $3 \cdot 13 \cdot 17 \cdot 31$ \\
\hline 209 & 6 & 37 & 2 & 4 & $2^{3} \cdot 3 \cdot 37$ & 451 & 22 & 235 & 4 & 8 & $2^{3} \cdot 5 \cdot 11 \cdot 47$ \\
\hline 210 & 15 & 915 & 1 & 8 & $3 \cdot 5 \cdot 61$ & 452 & 58 & 91 & 4 & 8 & $2^{3} \cdot 7 \cdot 13 \cdot 29$ \\
\hline 211 & 10 & 115 & 1 & 2 & $2^{3} \cdot 5 \cdot 23$ & 453 & 19 & 1155 & 8 & 32 & $3 \cdot 5 \cdot 7 \cdot 11 \cdot 19$ \\
\hline 212 & 2 & 115 & 2 & 2 & $2^{3} \cdot 5 \cdot 23$ & 454 & 43 & 130 & 4 & 8 & $2^{3} \cdot 5 \cdot 13 \cdot 43$ \\
\hline 213 & 7 & 33 & 4 & 8 & $2^{2} \cdot 3 \cdot 7 \cdot 11$ & 455 & 67 & 85 & 4 & 8 & $2^{2} \cdot 5 \cdot 17 \cdot 67$ \\
\hline 214 & 5 & 235 & 1 & 2 & $2^{2} \cdot 5 \cdot 47$ & 456 & 22 & 267 & 4 & 8 & $2^{3} \cdot 3 \cdot 11 \cdot 89$ \\
\hline 215 & 19 & 51 & 2 & 4 & $3 \cdot 17 \cdot 19$ & 457 & 37 & 163 & 2 & 2 & $2^{2} \cdot 37 \cdot 163$ \\
\hline 216 & 2 & 123 & 2 & 4 & $2^{3} \cdot 3 \cdot 41$ & 458 & 10 & 627 & 8 & 32 & $2^{5} \cdot 3 \cdot 5 \cdot 11 \cdot 19$ \\
\hline 217 & 13 & 19 & 2 & 2 & $2^{2} \cdot 13 \cdot 19$ & 459 & 43 & 595 & 4 & 8 & $5 \cdot 7 \cdot 17 \cdot 43$ \\
\hline 218 & 11 & 91 & 2 & 2 & $7 \cdot 11 \cdot 13$ & 460 & 67 & 403 & 2 & 2 & $13 \cdot 31 \cdot 67$ \\
\hline 219 & 15 & 67 & 2 & 2 & $3 \cdot 5 \cdot 67$ & 461 & 19 & 1435 & 4 & 8 & $5 \cdot 7 \cdot 19 \cdot 41$ \\
\hline 220 & 1 & 253 & 1 & 2 & $2^{2} \cdot 11 \cdot 23$ & 462 & 37 & 187 & 4 & 8 & $2^{2} \cdot 11 \cdot 17 \cdot 37$ \\
\hline 221 & 11 & 253 & 1 & 2 & $2^{2} \cdot 11 \cdot 23$ & 463 & 67 & 435 & 4 & 8 & $3 \cdot 5 \cdot 29 \cdot 67$ \\
\hline 222 & 6 & 43 & 2 & 2 & $2^{3} \cdot 3 \cdot 43$ & 464 & 163 & 187 & 2 & 2 & $11 \cdot 17 \cdot 163$ \\
\hline 223 & 2 & 258 & 1 & 4 & $2^{3} \cdot 3 \cdot 43$ & 465 & 43 & 715 & 4 & 8 & $5 \cdot 11 \cdot 13 \cdot 43$ \\
\hline 224 & 7 & 37 & 2 & 4 & $2^{2} \cdot 7 \cdot 37$ & 466 & 43 & 795 & 4 & 8 & $3 \cdot 5 \cdot 43 \cdot 53$ \\
\hline 225 & 2 & 133 & 2 & 4 & $2^{3} \cdot 7 \cdot 19$ & 467 & 67 & 555 & 4 & 8 & $3 \cdot 5 \cdot 37 \cdot 67$ \\
\hline 226 & 1 & 267 & 2 & 4 & $2^{2} \cdot 3 \cdot 89$ & 468 & 58 & 163 & 2 & 2 & $2^{5} \cdot 29 \cdot 163$ \\
\hline 227 & 3 & 273 & 2 & 8 & $2^{2} \cdot 3 \cdot 7 \cdot 13$ & 469 & 163 & 235 & 2 & 2 & $5 \cdot 47 \cdot 163$ \\
\hline 228 & 15 & 57 & 2 & 8 & $2^{2} \cdot 3 \cdot 5 \cdot 19$ & 470 & 37 & 267 & 4 & 8 & $2^{2} \cdot 3 \cdot 37 \cdot 89$ \\
\hline 229 & 7 & 163 & 1 & 1 & $7 \cdot 163$ & 471 & 67 & 595 & 4 & 8 & $5.7 \cdot 17.67$ \\
\hline 230 & 11 & 1155 & 2 & 8 & $3 \cdot 5 \cdot 7 \cdot 11$ & 472 & 163 & 267 & 2 & 2 & $3 \cdot 89 \cdot 163$ \\
\hline 231 & 7 & 301 & 1 & 4 & $2^{2} \cdot 7 \cdot 43$ & 473 & 187 & 235 & 4 & 8 & $5 \cdot 11 \cdot 17 \cdot 47$ \\
\hline 232 & 3 & 403 & 2 & 4 & $3 \cdot 13 \cdot 31$ & 474 & 123 & 403 & 4 & 8 & $3 \cdot 13 \cdot 31 \cdot 41$ \\
\hline 233 & 3 & 1227 & 1 & 2 & $3 \cdot 409$ & 475 & 187 & 267 & 4 & 8 & $3 \cdot 11 \cdot 17 \cdot 89$ \\
\hline 234 & 11 & 1243 & 1 & 2 & $11 \cdot 113$ & 476 & 123 & 427 & 4 & 8 & $3 \cdot 7 \cdot 41 \cdot 61$ \\
\hline 235 & 11 & 115 & 2 & 4 & $5 \cdot 11 \cdot 23$ & 477 & 43 & 1435 & 4 & 8 & $5 \cdot 7 \cdot 41 \cdot 43$ \\
\hline 236 & 19 & 67 & 1 & 1 & $19 \cdot 67$ & 478 & 19 & 3315 & 8 & 32 & $3 \cdot 5 \cdot 13 \cdot 17 \cdot 19$ \\
\hline 237 & $\overline{3}$ & 427 & $\overline{2}$ & 4 & $3 \cdot 7 \cdot 61$ & 479 & 163 & 403 & 2 & 2 & $13 \cdot 31 \cdot 163$ \\
\hline 238 & 7 & 322 & 1 & 4 & $2^{3} \cdot 7 \cdot 23$ & 480 & 163 & 427 & 2 & 4 & $7 \cdot 61 \cdot 163$ \\
\hline 239 & $\overline{7}$ & 187 & 2 & 2 & $7 \cdot 11 \cdot 17$ & 481 & 235 & 427 & 4 & 8 & $5 \cdot 7 \cdot 47 \cdot 61$ \\
\hline 240 & 10 & 330 & 1 & 8 & $2^{3} \cdot 3 \cdot 5 \cdot 11$ & 482 & 43 & 3315 & 8 & 32 & $3 \cdot 5 \cdot 13 \cdot 17 \cdot 43$ \\
\hline
\end{tabular}


The fields $N_{4}=\mathbb{Q}\left(\sqrt{-m_{1}}, \sqrt{-m_{2}}\right)$ with $\exp \left(\mathrm{Cl}\left(N_{4}\right)\right) \leqslant 2$.

\begin{tabular}{|c|c|c|c|c|c||c|c|c|c|c|c|}
\hline$N_{r}$ & $m_{1}$ & $m_{2}$ & $h_{N_{4}^{+}}$ & $h_{N_{4}}$ & $f_{N_{4}}$ & $N_{r}$ & $m_{1}$ & $m_{2}$ & ${ }^{h} N_{4}^{+}$ & $h_{N_{4}}$ & $f_{N_{4}}$ \\
\hline 241 & 2 & 330 & 2 & 8 & $2^{3} \cdot 3 \cdot 5 \cdot 11$ & 483 & 163 & 1435 & 4 & 8 & $5 \cdot 7 \cdot 41 \cdot 163$ \\
\hline 242 & $1 !$ & 330 & 2 & 8 & $2^{3} \cdot 3 \cdot 5 \cdot 11$ & & & & & & \\
\hline
\end{tabular}

Table 5

The fields $N_{8}=\mathbb{Q}\left(\sqrt{-m_{1}}, \sqrt{-m_{2}}, \sqrt{-m_{3}}\right)$ with $\exp \left(\mathrm{Cl}\left(N_{8}\right)\right) \leqslant 2$.

\begin{tabular}{|c|c|c|c|c|c|c|c|c|c|c|c|c|c|}
\hline Nr. & $m_{l}$ & $m_{2}$ & $m_{3}$ & ${ }^{h_{N_{8}^{+}}}$ & $h_{N_{8}}$ & $f_{N_{B}}$ & $\mathrm{Nr}$. & $\overline{m_{1}}$ & $m_{2}$ & $\overrightarrow{m s}$ & ${ }^{h} \mathrm{~N}^{+}$ & $\overline{h_{N_{8}}}$ & $f_{N_{B}}$ \\
\hline 1 & 1 & 2 & 3 & 1 & 1 & $2^{3} \cdot 3$ & 51 & 3 & 11 & 51 & 1 & 1 & $3 \cdot 11 \cdot 17$ \\
\hline 2 & 1 & 2 & 5 & 1 & 1 & $2^{3} \cdot 5$ & 52 & 2 & 7 & 11 & 1 & 2 & $2^{5} \cdot 7 \cdot 11$ \\
\hline 3 & 1 & 2 & 7 & 1 & 2 & $2^{3} \cdot 7$ & 53 & 7 & 11 & 14 & 1 & 2 & $2^{3} \cdot 7 \cdot 11$ \\
\hline 4 & 1 & 3 & 5 & 1 & 1 & $2^{2} \cdot 3 \cdot 5$ & 54 & I & 7 & 22 & 2 & $B$ & $2^{3} \cdot 7 \cdot 11$ \\
\hline 5 & 1 & 3 & 7 & 1 & 1 & $2^{2} \cdot 3 \cdot 7$ & 55 & 2 & 7 & 22 & 2 & 8 & $2^{3} \cdot 7 \cdot 11$ \\
\hline 6 & 1 & 2 & 11 & 1 & 1 & $2^{3} \cdot 11$ & 56 & 3 & 11 & 19 & 1 & 1 & $3 \cdot 11 \cdot 19$ \\
\hline 7 & 3 & 7 & 15 & 1 & 1 & $3 \cdot 5 \cdot 7$ & 37 & 1 & 11 & 15 & 2 & 8 & $2^{2} \cdot 3 \cdot 5 \cdot 11$ \\
\hline 8 & 2 & 3 & 10 & ? & 1 & $2^{3} \cdot 3 \cdot 5$ & 38 & 1 & 11 & 17 & 1 & 2 & $2^{2} \cdot 11 \cdot 17$ \\
\hline 9 & 3 & 6 & 15 & 1 & 2 & $2^{3} \cdot 3 \cdot 5$ & 59 & 2 & 3 & 19 & 2 & 4 & $2^{5} \cdot 5 \cdot 19$ \\
\hline 10 & 3 & 5 & 6 & 2 & 4 & $2^{3} \cdot 3 \cdot 5$ & 60 & 1 & 10 & 19 & 2 & 4 & $2^{5} \cdot 5 \cdot 19$ \\
\hline 11 & 1 & 3 & 11 & 1 & 1 & $2^{2} \cdot 3 \cdot 11$ & 61 & 1 & 13 & 15 & 2 & 8 & $2^{2} \cdot 3 \cdot 5 \cdot 13$ \\
\hline 12 & 1 & 2 & 17 & 1 & 4 & $2^{3} \cdot 17$ & 62 & 3 & 5 & 13 & 2 & 8 & $2^{2} \cdot 3 \cdot 5 \cdot 13$ \\
\hline 13 & 1 & 5 & 7 & 1 & 1 & $2^{2} \cdot 5 \cdot 7$ & 63 & 10 & 15 & 35 & 1 & 8 & $2^{3} \cdot 3 \cdot 5 \cdot 7$ \\
\hline 14 & 1 & 3 & 13 & 1 & 2 & $2^{2} \cdot 3 \cdot 13$ & 64 & 3 & 6 & 35 & 2 & 8 & $2^{5} \cdot 3 \cdot 5 \cdot 7$ \\
\hline 15 & 3 & 11 & 15 & 1 & 2 & $3 \cdot 5 \cdot 11$ & 65 & 1 & 3 & 73 & 2 & 8 & $2^{2} \cdot 3 \cdot 73$ \\
\hline 16 & 2 & 3 & 7 & l & 1 & $2^{3} \cdot 3 \cdot 7$ & 66 & 3 & 19 & 51 & 1 & 2 & $3 \cdot 17 \cdot 19$ \\
\hline 17 & 3 & 6 & 7 & 1 & 2 & $2^{3} \cdot 3 \cdot 7$ & 67 & 2 & 3 & 82 & 1 & 2 & $2^{3} \cdot 3 \cdot 41$ \\
\hline 18 & 1 & 2 & 21 & 1 & 4 & $2^{3} \cdot 3 \cdot 7$ & 68 & 3 & 5 & 51 & 2 & 8 & $2^{2} \cdot 3 \cdot 5 \cdot 17$ \\
\hline 18 & 2 & 6 & 7 & 2 & 4 & $2^{3} \cdot 3 \cdot 7$ & 69 & 2 & 3 & 43 & 1 & 2 & $2^{3} \cdot 3 \cdot 43$ \\
\hline 20 & 1 & 6 & 7 & 2 & 4 & $2^{3} \cdot 3 \cdot 7$ & 70 & 1 & 7 & 37 & 1 & 2 & $2^{2} \cdot 7 \cdot 37$ \\
\hline 21 & 3 & 6 & 21 & $\mathrm{I}$ & 4 & $2^{3} \cdot 3 \cdot 7$ & 71 & 1 & 3 & 91 & 2 & 8 & $2^{2} \cdot 3 \cdot 7 \cdot 13$ \\
\hline 22 & 1 & 3 & 17 & 1 & 2 & $2^{2} \cdot 3 \cdot 17$ & 72 & 5 & 15 & 19 & 2 & $B$ & $2^{2} \cdot 3 \cdot 3 \cdot 19$ \\
\hline 23 & 1 & 5 & 11 & 1 & 2 & $2^{2} \cdot 5 \cdot 11$ & 73 & 1 & 7 & 43 & 1 & 2 & $2^{2} \cdot 7 \cdot 43$ \\
\hline 24 & 1 & $\overline{3}$ & 19 & 1 & 1 & $2^{2} \cdot 3 \cdot 19$ & 74 & 1 & 17 & 19 & 2 & g & $2^{2} \cdot 17 \cdot 19$ \\
\hline 25 & 3 & 6 & 11 & I & 1 & $2^{3} \cdot 3 \cdot 11$ & 75 & 3 & 10 & 11 & 2 & 8 & $2^{5} \cdot 3 \cdot 5 \cdot 11$ \\
\hline 26 & 2 & 3 & 11 & 1 & 2 & $2^{3} \cdot 3 \cdot 11$ & 76 & 2 & 11 & 15 & 2 & 8 & $2^{5} \cdot 3 \cdot 5 \cdot 11$ \\
\hline 27 & 2 & 6 & 11 & 2 & 4 & $2^{3} \cdot 3 \cdot 11$ & 77 & 7 & 15 & 91 & 2 & 8 & $3 \cdot 5 \cdot 7 \cdot 13$ \\
\hline 28 & 2 & 3 & 22 & 2 & 4 & $2^{3} \cdot 3 \cdot 11$ & 78 & 2 & 11 & 34 & 1 & 2 & $2^{3} \cdot 11 \cdot 17$ \\
\hline 29 & 1 & 6 & 22 & 1 & 4 & $2^{3} \cdot 3 \cdot 11$ & 79 & 5 & 7 & 11 & 2 & 8 & $2^{2} \cdot 5 \cdot 7 \cdot 11$ \\
\hline 30 & 3 & 7 & 39 & i & 2 & $3 \cdot 7 \cdot 13$ & 80 & 1 & 11 & 35 & 2 & 8 & $2^{2} \cdot 5 \cdot 7 \cdot 11$ \\
\hline 31 & 2 & 7 & 10 & 1 & 1 & $2^{3} \cdot 5 \cdot 7$ & 81 & 1 & 21 & 57 & 1 & 8 & $2^{2} \cdot 3 \cdot 7 \cdot 19$ \\
\hline 32 & 2 & 5 & 7 & 2 & 4 & $2^{3} \cdot 5 \cdot 7$ & 82 & 2 & 7 & 58 & I & 2 & $2^{3} \cdot 7 \cdot 29$ \\
\hline 33 & 1 & 7 & 10 & 2 & 4 & $2^{3} \cdot 5 \cdot 7$ & 83 & 2 & 11 & 19 & 1 & 2 & $2^{3} \cdot 11 \cdot 19$ \\
\hline 34 & 1 & 2 & 35 & 2 & 4 & $2^{3} \cdot 5 \cdot 7$ & 84 & 2 & 7 & 33 & 4 & 32 & $2^{3} \cdot 3 \cdot 7 \cdot 11$ \\
\hline 35 & 5 & 10 & 35 & 1 & 4 & $2^{3} \cdot 5 \cdot 7$ & 83 & 7 & 15 & 19 & 2 & 8 & $3 \cdot 5 \cdot 7 \cdot 19$ \\
\hline 36 & 1 & 7 & 11 & 1 & 2 & $2^{2} \cdot 7 \cdot 11$ & 86 & $\mathbf{3}$ & 19 & 35 & 2 & 8 & $3 \cdot 5 \cdot 7 \cdot 19$ \\
\hline 37 & 1 & 6 & 13 & 1 & 4 & $2^{3} \cdot 3 \cdot 13$ & 87 & 3 & 11 & 185 & 2 & 8 & $3 \cdot 5 \cdot 11 \cdot 13$ \\
\hline 38 & 2 & 3 & 13 & 2 & 4 & $2^{3} \cdot 3 \cdot 13$ & 88 & 6 & 7 & 78 & 2 & 8 & $2^{5} \cdot 3 \cdot 7 \cdot 13$ \\
\hline 39 & 1 & 7 & 13 & 1 & 1 & $2^{2} \cdot 7 \cdot 13$ & 89 & 1 & 15 & 37 & 2 & 8 & $2^{2} \cdot 3 \cdot 5 \cdot 37$ \\
\hline 40 & 7 & 11 & 35 & i & 2 & $5 \cdot 7 \cdot 11$ & 90 & 3 & 5 & 37 & 2 & 8 & $2^{2} \cdot 3 \cdot 5 \cdot 37$ \\
\hline 41 & 3 & 6 & 51 & 1 & 2 & $2^{3} \cdot 3 \cdot 17$ & 91 & 1 & 33 & 51 & 2 & 8 & $2^{2} \cdot 3 \cdot 11 \cdot 17$ \\
\hline 42 & 2 & 3 & 34 & 1 & 2 & $2^{3} \cdot 3 \cdot 17$ & 92 & 5 & 21 & 13 & 2 & 8 & $2^{2} \cdot 5 \cdot 11 \cdot 13$ \\
\hline 43 & 1 & 6 & 17 & 1 & 8 & $2^{3} \cdot 3 \cdot 17$ & 93 & 11 & 22 & 35 & 2 & 8 & $2^{5} \cdot 5 \cdot 7 \cdot 11$ \\
\hline 44 & 1 & 2 & 51 & 4 & 8 & $2^{3} \cdot 3 \cdot 17$ & 94 & 3 & 51 & 193 & 2 & 16 & $3 \cdot 5 \cdot 13 \cdot 17$ \\
\hline 45 & 2 & 6 & 34 & 1 & 8 & $2^{3} \cdot 3 \cdot 17$ & 95 & 11 & 19 & 187 & 1 & 2 & $11 \cdot 17 \cdot 19$ \\
\hline 46 & 2 & 10 & 11 & 1 & 2 & $2^{3} \cdot 5 \cdot 11$ & 96 & 5 & 11 & 85 & 2 & 8 & $2^{2} \cdot 5 \cdot 11 \cdot 17$ \\
\hline 47 & 5 & 10 & 11 & 2 & 4 & $2^{3} \cdot 5 \cdot 11$ & 97 & 7 & 19 & 70 & 2 & 8 & $2^{3} \cdot 5 \cdot 7 \cdot 19$ \\
\hline 48 & 2 & $\mathbf{3}$ & 19 & $\frac{2}{1}$ & 2 & $2^{3} \cdot 3 \cdot 19$ & 98 & 3 & 13 & 37 & 4 & 32 & $2^{2} \cdot 3 \cdot 13 \cdot 37$ \\
\hline 49 & 2 & 6 & 19 & 2 & 4 & $2^{3} \cdot 3 \cdot 19$ & 99 & 5 & 19 & 85 & 4 & 32 & $2^{2} \cdot 5 \cdot 17 \cdot 19$ \\
\hline 50 & 1 & 7 & 19 & 1 & 1 & $2^{2} \cdot 7 \cdot 19$ & & & & & & & \\
\hline
\end{tabular}


Table 6

The fields $N_{16}=\mathbb{Q}\left(\sqrt{-m_{1}}, \sqrt{-m_{2}}, \sqrt{-m_{3}}, \sqrt{-m_{4}}\right)$ with $\exp \left(\mathrm{Cl}\left(N_{16}\right)\right) \leqslant 2$.

\begin{tabular}{|c|c|c|c|c|c|c|c|}
\hline$N r$. & $m_{1}$ & $m_{2}$ & $m_{3}$ & $m_{4}$ & $h_{N_{16}^{+}}$ & $h_{N_{16}}$ & $f_{N_{16}}$ \\
\hline 1 & 1 & 2 & 3 & 5 & 1 & 2 & $2^{3} \cdot 3 \cdot 5$ \\
\hline 2 & 1 & 3 & 5 & 7 & 1 & 4 & $2^{2} \cdot 3 \cdot 5 \cdot 7$ \\
\hline 3 & 2 & 3 & 7 & 10 & 1 & 4 & $2^{3} \cdot 3 \cdot 5 \cdot 7$ \\
\hline
\end{tabular}

\section{REFERENCES}

[1] C. Batut, K. Belabas, D. Bernardi, H. Cohen and M. Oliver, PARI-GP version 2.1.5.

[2] Z.I. Borevich and I.R. Shafarevich, Number theory (Academic Press, New York and London, 1966).

[3] D.W. Boyd and H. Kisilevsky, 'On the exponent of the ideal class groups of complex quadratic fields', Proc. Amer. Math. Soc. 31 (1972), 433-436.

[4] J.W.S. Cassels and A. Fröhlich, Algebraic number theory (Academic Press, London, New York, 1967).

[5] K.-Y. Chang and S.-H. Kwon, 'The imaginary abelian number fields with class numbers equal to their genus class numbers', J. Theor. Nombres Bordeaux 12 (2000), 349-365.

[6] S. Chowla, 'An extension of Heilbronn's class-number theorem', Quart. J. Math. Oxford Ser. 25 (1934), 304-307.

[7] P.E. Conner and J. Hurrelbrink, 'Class number parity', Series in Pure Mathematics 8 (World Scientific, Singapore).

[8] D.A. Cox, Primes of the form $x^{2}+n y^{2}$ (John Wiley \& Sons, Inc., 1989).

[9] M. Daberkow, C. Fieker, J. Klüners, M. Phost, K. Roegner and K. Wildanger, 'Computational algebra and number theory, KANT V4', J. Symbolic Comp. 24 (1997), 267-283.

[10] A.G. Earnest, 'Exponents of the class groups of imaginary abelian number fields', Bull. Austral. Math. Soc. 35 (1987), 231-245.

[11] A.G. Earnest and O.H. Körner, 'On ideal class groups of 2-power exponent', Proc. Amer. Math. Soc. 36 (1982), 196-198.

[12] K. Horie, 'On the exponents of ideal class groups of cyclotomic fields', Proc. Amer. Math. Soc. 119 (1993), 1049-1052.

[13] K. Horie and M. Horie, 'CM-fields and exponents of their ideal class groups', Acta Arith. 55 (1990), 157-170.

[14] K. Horie and M. Horie, 'On the exponents of ideal class groups of CM-fields', Lecture Notes in Math. 1434 (1990), 143-148.

[15] K. Horie and M. Horie, 'On the 2-class groups of cyclotomic fields whose maximal real subfields have odd class numbers', Proc. Amer. Math. Soc. 123 (1995), 2643-2649.

[16] S. Louboutin, 'Minoration(sous l'hypothèse de Riemann généralisée) des nombres de classes des corps quadratiques imaginaires. Application', C. R. Acad. Sci. Paris 310 (1990), 795-800.

[17] S. Louboutin, 'Determination of all nonquadratic imaginary cyclic number fields of 2 -power degrees with ideal class groups of exponents $\leqslant 2$ ', Math. Comp. 64 (1995), $323-340$. 
[18] S. Louboutin, 'Determination of all quaternion CM-fields with ideal class groups of exponent 2', Osaka J. Math. 36 (1999), 229-257.

[19] S. Louboutin, H.-S. Yang and S.-H. Kwon, 'The non-normal quartic CM-fields and the dihedral octic CM-fields with ideal class group of exponent $\leqslant 2$, Math. Slovaca (to appear).

[20] I. Miyada, 'On imaginary abelian number fields of type $(2, \cdots, 2)$ with one class in each genus', Manuscripta Math. 88 (1995), 535-540.

[21] I. Niven, H.S. Zuckerman and H.L. Montgomery, An introduction to the theory of numbers, (fifth edition) (John Wiley \& Sons, Inc., 1991).

[22] F. Pappalardi, 'On the exponent of the ideal class group of $\mathbb{Q}(\sqrt{-d})$ ', Proc. Amer. Math. Soc. 123 (1995), 663-671.

[23] L. Washington, Introduction to cyclotomic fields (2nd edition), Graduate texts in Mathematics 83 (Springer-Verlag, New York, 1997).

[24] P.J. Weinberger, 'Exponents of the class groups of complex quadratic fields', Acta Arith. 22 (1973), 117-124.

Department of Mathematics Education

Korea University

136-701, Seoul

Korea

e-mail: jh-ahn@korea.ac.kr

sounhikwon@korea.ac.kr 